\title{
Mutual Coupling Reduction Using Improved Dual-Layer Mushroom and E-Shaped Stub
}

\author{
Chunxia Cheng ${ }^{1}$ and Liyan Luo ${ }^{2}$ \\ ${ }^{1}$ School of Aerospace Science and Technology, Xidian University, Xi'an 710071, China \\ ${ }^{2}$ Provincial Ministry of Education Key Laboratory of Cognitive Radio and Signal Processing, \\ Guilin University of Electronic Technology, Guilin, Guangxi 541004, China \\ Correspondence should be addressed to Chunxia Cheng; cxcheng@xidian.edu.cn
}

Received 29 September 2020; Revised 5 January 2021; Accepted 1 February 2021; Published 19 February 2021

Academic Editor: Giuseppe Castaldi

Copyright (c) 2021 Chunxia Cheng and Liyan Luo. This is an open access article distributed under the Creative Commons Attribution License, which permits unrestricted use, distribution, and reproduction in any medium, provided the original work is properly cited.

\begin{abstract}
The improved dual-layer mushroom (IDLM) and back-to-back E-shaped stubs for mutual coupling reduction between microstrip patch antennas are presented in this paper. The IDLM unit consists of one upper layer complementary split-ring resonator lattice and four lower layer lattices, whose centers are connected to the ground by a pin. The decoupling structure can prevent the surface current from one antenna port to another, so as to improve the isolation between the antennas. The proposed antenna works in the open wireless communication band of $2.45 \mathrm{GHz}$. Using the proposed decoupling structure, a low mutual coupling level ranging from -27 to $-40 \mathrm{~dB}$ is obtained when the center distance of the adjacent patches is $0.5 \lambda_{0}$. The total size of the decoupling antenna is $99 \times 41 \times 2.4 \mathrm{~mm}^{3}$ with a frequency range of $2.42-2.48 \mathrm{GHz}$ for $S_{11}<-10 \mathrm{~dB}$. The proposed decoupling structure can also improve the average gain and efficiency of the antenna by $0.1 \mathrm{~dB}$ and $5 \%$, respectively. The antenna is studied from the aspects of isolation, return loss, current and electric field distribution, radiation pattern, and diversity performance. The designed decoupling antenna is fabricated and measured. The pattern, isolation, and return loss of the tested results show good consistence with the simulation results. The diversity gain and envelop correlation coefficient of the diversity performance show that the designed antenna can be used in MIMO or Rx/Tx systems.
\end{abstract}

\section{Introduction}

In multiantenna applications such as multiple input multiple output (MIMO) system, Rx/Tx system, and array antenna, the coupling between neighboring antenna elements is an important problem that cannot be ignored. The mutual coupling (MC) between antennas will result in low antenna efficiency, poor impedance matching, and radiation performance. Thus, reducing MC involves various types of antennas, such as 3D nonplanar antenna array [1], waveguide slot array [2], and microstrip patch antenna [3]. As one of the most commonly used antennas, microstrip antenna is extensively employed in many wireless systems. Therefore, there are many literatures on the decoupling method of microstrip antenna. The decoupling structure can be placed on the upper layer of the microstrip antenna [4] or between the antenna elements [5].
In previous studies, several techniques have been proposed to enhance the isolation between adjacent microstrip antenna elements. Common decoupling technologies include lumped elements decoupling network $[6,7]$, resonator [8], defected grounded structures (DGSs) [9, 10], polarization-conversion isolator [11], electromagnetic bandgap [12-14], and metamaterials [15-17]. The use of DGS can suppress the surface wave and improve isolation. However, this method often leads to a lower front-to-back ratio, as mentioned in $[9,10]$. The combination of one-dimensional split ring resonator (SRR) and electromagnetic bandgap (EBG) [12], Z-shaped etched metallic elements [13], and fractal-based EBG [14] is adopted to reduce the MC. In [15], the metamaterial is introduced on both the bottom and top sides of the antenna array to enhance the isolation of $14 \mathrm{~dB}$, but the results indicate a poor front-to-back ratio. Elliptical SRRs have been placed between the ground plane and the 
patch to achieve $44 \mathrm{~dB}$ isolation [16]. About $35 \mathrm{~dB}$ isolation was achieved by using flower-shaped metamaterial [17].

In this paper, the MC is reduced by embedding duallayer mushroom (IDLM) and back-to-back E-shaped stubs between the two microstrip antennas. The IDLM element is improved on the basis of double-layer rectangular EBG [18], which has a smaller size than simple double-layer EBG. The combination of IDLM and E-shaped stub reduces the MC by $19 \mathrm{~dB}$ at the working frequency of the antenna. The envelope correlation coefficient (ECC) of the designed antenna is low, the diversity gain $(D G)$ is high, and the total active reflection coefficient (TARC) is $-11 \mathrm{~dB}$ at $2.45 \mathrm{GHz}$, which is available for MIMO or Rx/Tx system. The structure of the proposed antenna geometry and $\mathrm{MC}$ reduction performances are shown in Section 2. The patterns, $S$-parameters, ECC, DG, and TARC of the antenna are indicated in Section 3. Finally, Section 4 gives the design conclusion.

\section{Antenna Design and Analysis}

2.1. Structure of Antenna. The structure and geometry of the designed antenna are provided in Figure 1(a). The substrate of the antenna is FR4 with loss tangent $(\delta)$ of 0.02 , and the total size of the antenna is $99 \times 41 \times 2.4 \mathrm{~mm}^{3}$. It consists of four IDLM elements and two back-to-back E-shaped stubs, which are located between the two antenna elements.

The resonant frequency of the designed antenna is about $2.45 \mathrm{GHz}$. To obtain this resonance frequency, the patch antenna dimensions are as follows: $L_{p}=24.4 \mathrm{~mm}$ and $W_{p}=27.4 \mathrm{~mm}$. The two antenna elements are fed by a coaxial probe, respectively. The center-to-center spacing $\left(S_{1}\right)$ of the element is $64.1 \mathrm{~mm}$, which is equivalent to half of the wavelength corresponding to $2.45 \mathrm{GHz}$.

To reduce the surface current flow and the MC between the array radiating elements, back-to-back E-shaped stubs and an array of IDLM have been implemented between the radiating elements. The IDLM units consist of a double-layer split-ring resonator with a similar structure shown in Figure 1(b). The lower layer consists of four elements of the same size, and the upper layer is an element of another size. The resistance band of the IDLM is used to prevent the electromagnetic wave transmitting to the nonexcitation element. The specific parameters of the optimized IDLM are shown in Table 1 . The length of $L_{1}+W_{1} / 2-W_{3}$ in the E-shaped stub is about one-fourth of the effective wavelength corresponding to $2.45 \mathrm{GHz}$. The E-shaped stub can resonate at $2.45 \mathrm{GHz}$ which is the center frequency of the antenna, so it can effectively prevent the electromagnetic wave from coupling to the adjacent antenna element. Figure 2 shows the relationship between the length of E-shaped stubs and $S_{21}$ of the adjacent antenna elements. $W_{1}$ remains unchanged at $27.4 \mathrm{~mm}$ when $L_{1}$ changed, and $L_{1}$ remains unchanged at $5.5 \mathrm{~mm}$ when $W_{1}$ changed. It can be seen from Figure 2 that, with the increase in $L_{1}$ or $W_{1}$, the frequency with a smaller $S_{21}$ value gradually decreases because the increase in $L_{1}$ or $W_{1}$ leads to the decrease in the resonance frequency. By adjusting $L_{1}$ or $W_{1}$, the isolation of the required frequency band can be improved. The optimized parameters of the E-shaped stubs are shown in Table 1.
Next, the position of the E-shaped stub is explored. Figure 3(a) shows the $S$-parameter results variation for various spacing $\left(S_{2}\right)$ between the E-shaped stub and IDLM. $S_{3}$ remains unchanged at $7.2 \mathrm{~mm}$, and the other parameters of the antenna are listed in Table 1 when $S_{2}$ changed. As $S_{2}$ increases from 1 to $3 \mathrm{~mm}$ and then $6 \mathrm{~mm}$, the $S_{21}$ decreases from $-23.7 \sim-27.4$ to $-24.9 \sim-36.2 \mathrm{~dB}$ and then increases to $-24.5 \sim 35.1 \mathrm{~dB}$. The resonate frequency of the antenna increases with the increase in $S_{2}$. The best $S$-parameter is obtained when $S_{2}=3 \mathrm{~mm}$. Figure 3(b) shows the effect of E-shaped stub offset $\left(S_{3}\right)$ on the decoupling performance. $S_{2}$ remains unchanged at $3 \mathrm{~mm}$, and the other parameters are as Table 1 when $S_{3}$ changed. When $S_{3}=1.2$ and $13.2 \mathrm{~mm}$, that is, the E-shaped stub offsets too far from the center of the radiation patch, the frequency of $S_{21}$ minimum becomes higher. This is because the effective length of the E-shaped stub becomes smaller. However, when $S_{3}$ increases from 3.2 to $11.2 \mathrm{~mm}$, the $S_{21}$ remains almost unchanged in the operating frequency band of the antenna.

Figure 4 illustrates the $S$-parameter results versus frequency for the various center-to-center spacing $\left(S_{1}\right)$. As $S_{1}$ reduces from $64.1 \mathrm{~mm}$ to $56.1 \mathrm{~mm}$, the isolation is less affected, while the frequency corresponding to the minimum value of $S_{11}$ increases.

2.2. Coupling Reduction Performance. The configurations of the three antennas are presented in Figure 5: (1) AT1 is a dual conventional rectangular antenna without decoupling structure, (2) AT2 is a dual antenna with an array of IDLM, and (3) AT3 is a dual antenna with back-to-back E-shaped stubs on both sides of the IDLM. Figure 6 illustrates the transmission coefficient $\left(S_{21}\right)$ and simulated reflection coefficient $\left(S_{11}\right)$ of the abovementioned three antennas. It is observed that AT1 has poor isolation. The MC of AT2 is slightly lower than that of AT1 because there are fewer IDLM elements in a limited space. The MC of AT3 is obviously improved by printing E-shaped stubs on both sides of the IDLM. The minimum coupling degree is reduced by approximately $20 \mathrm{~dB}$, which is less than $-27 \mathrm{~dB}$ in the entire working bandwidth, as illustrated in Figure 6.

To express the reason for the increased isolation, the surface current distribution of AT1, AT2, and AT3 at $2.45 \mathrm{GHz}$ is shown in Figure 7. In the analysis, port 1 is excited and port 2 is loaded with a $50 \Omega$ standard resistance. As shown in Figure 7(a), before loading decoupling structure, the current induced by the right element is high when the left unit is excited. Therefore, the MC between elements of AT1 is high. The MC is reduced when IDLM is employed. As illustrated in Figure 7(b), the current on the right element decreases, but a part of current is still coupled to the right element. When introducing E-shaped stubs on both sides of the IDLM, most of the current is concentrated on the E-shaped stubs, resulting in a small-induced current on the right element, as illustrated in Figure $7(\mathrm{c})$. Therefore, the isolation is greatly improved.

To further analyze the influence of decoupling structure on antenna performance, the electric field without or with the decoupling structure is shown in Figure 8. The electric 


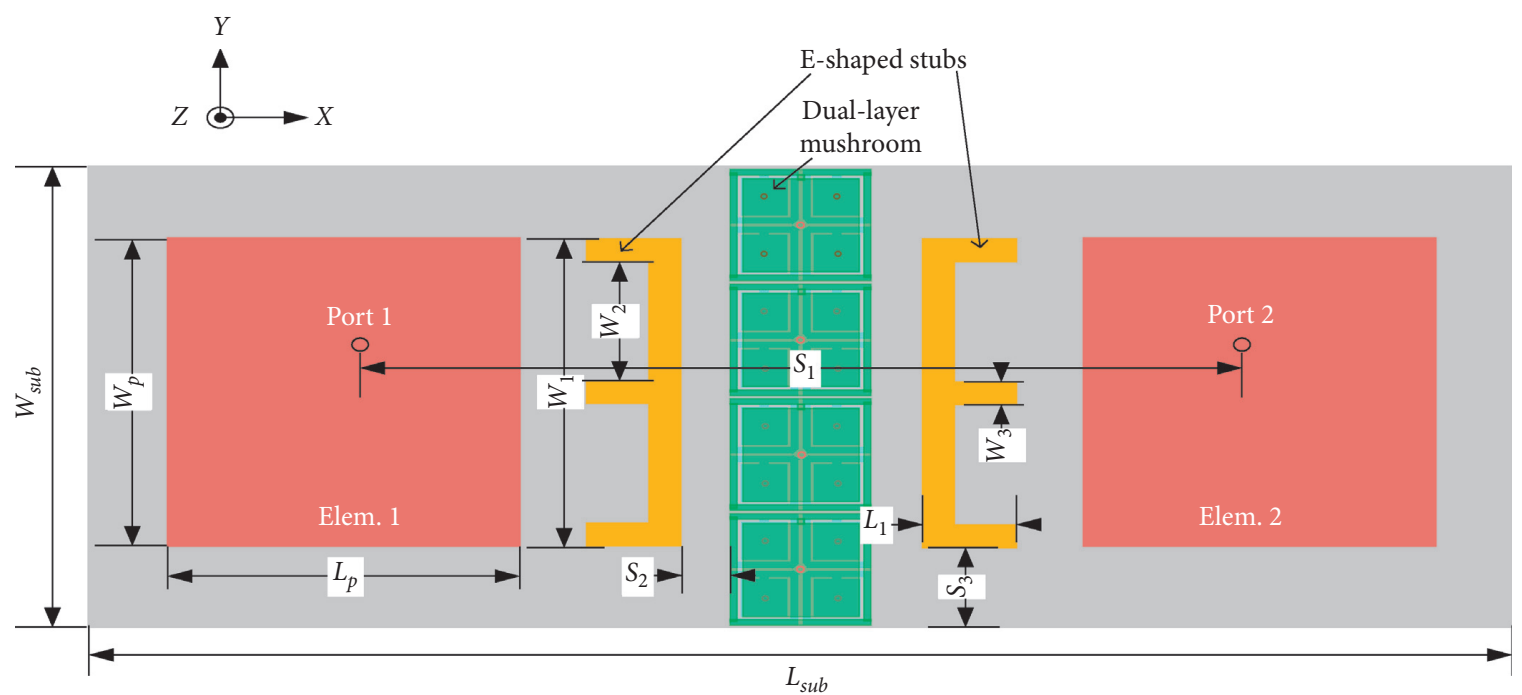

(a)

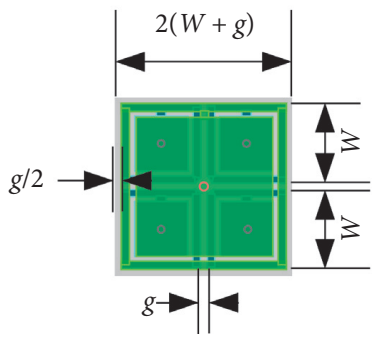

Top view

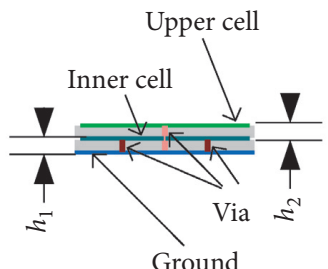

Side view

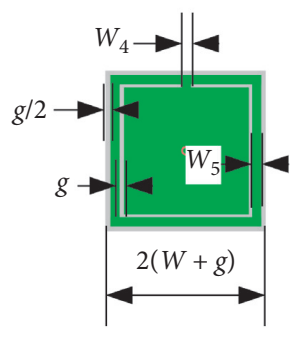

Upper cell

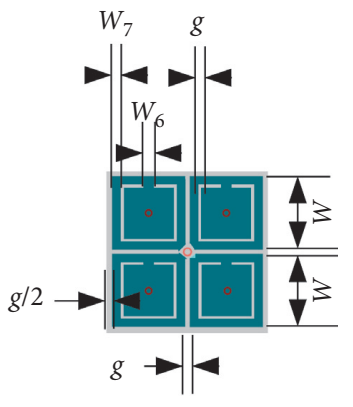

Inner cell

(b)

Figure 1: Geometry of the proposed antenna: (a) top view; (b) dual-layer mushroom cell.

TABle 1: Dimensions of optimized parameters of the proposed antenna.

\begin{tabular}{lccc}
\hline Parameters & Dimension $(\mathrm{mm})$ & Parameters & Dimension $(\mathrm{mm})$ \\
\hline$W_{1}$ & 27.4 & $h_{1}$ & 1.2 \\
$W_{2}$ & 10.7 & $h_{2}$ & 1.2 \\
$W_{3}$ & 2 & $W_{4}$ & 0.6 \\
$L_{1}$ & 5.5 & $W_{5}$ & 0.5 \\
$W$ & 4.8 & $W_{6}$ & 0.6 \\
$g$ & 0.3 & $W_{7}$ & 0.5 \\
\hline
\end{tabular}

field of excitation element is almost unaffected by the decoupling structure. But the electric field of nonexcited element rotates about 45 degrees. Therefore, the far-field pattern radiated by the right element has almost no effect on the copolarization pattern of the excited element. That is why the copolarization pattern of the excited element is not affected by the nonexcited element with the decoupling structure introduced. It can also be seen from the figure that, with introducing the decoupling structure, the feed point of the nonexcited element is at the minimum position of the electric field, so the feed port isolation between the two elements is improved.

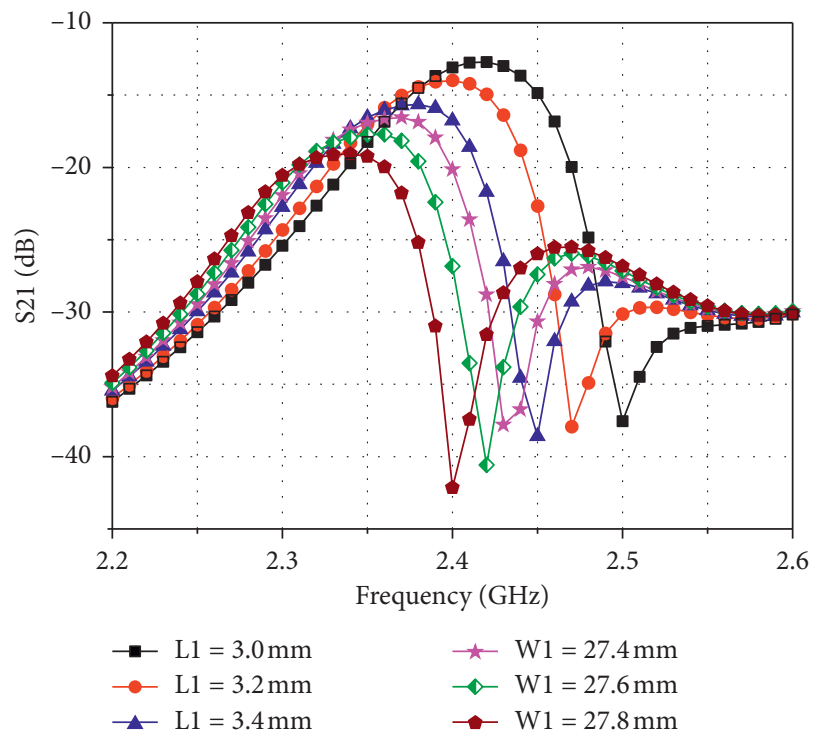

FIgURE 2: Relationship between E-shaped stub parameters and $S_{21}$.

The proposed decoupling structure in this paper is also effective for PIFAs shown in Figure 9. The $S$-parameters of 2-element PIFAs with and without the proposed decoupling 


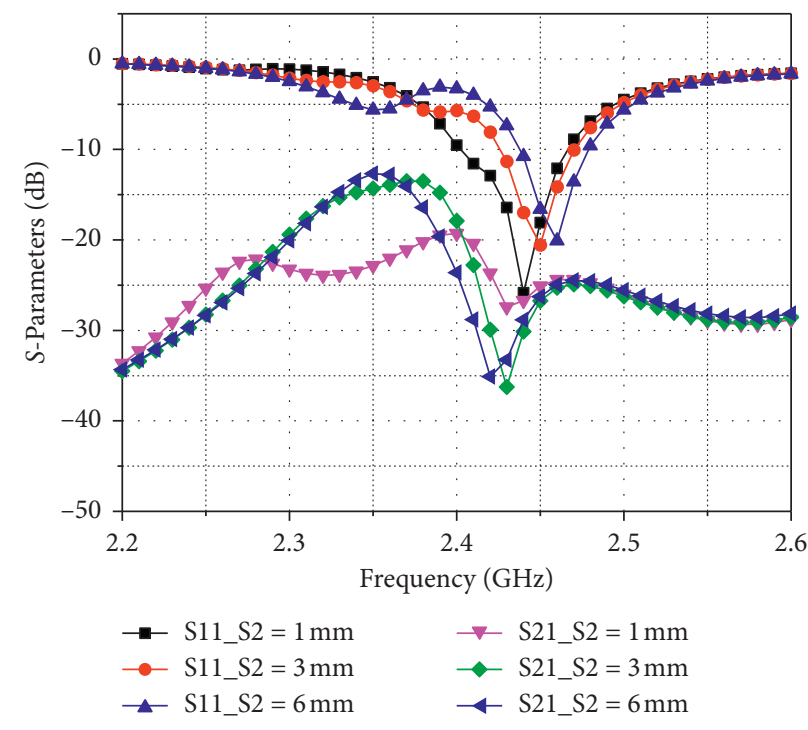

(a)

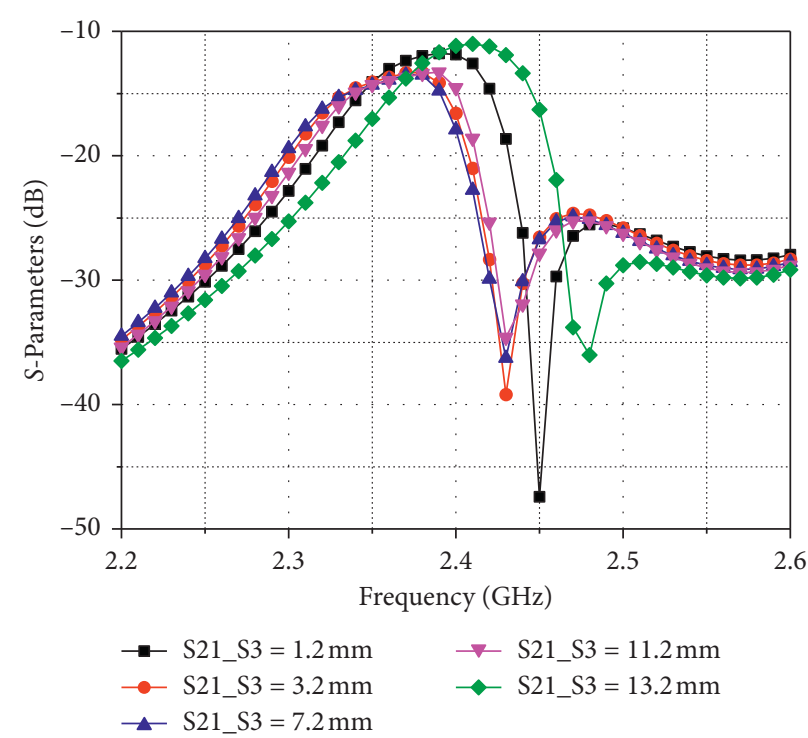

(b)

FIgURE 3: Comparison between the position of the E-shaped stub and S-parameters: (a) spacing between the E-shaped stub and IDLM; (b) the offset of E-shaped stub.

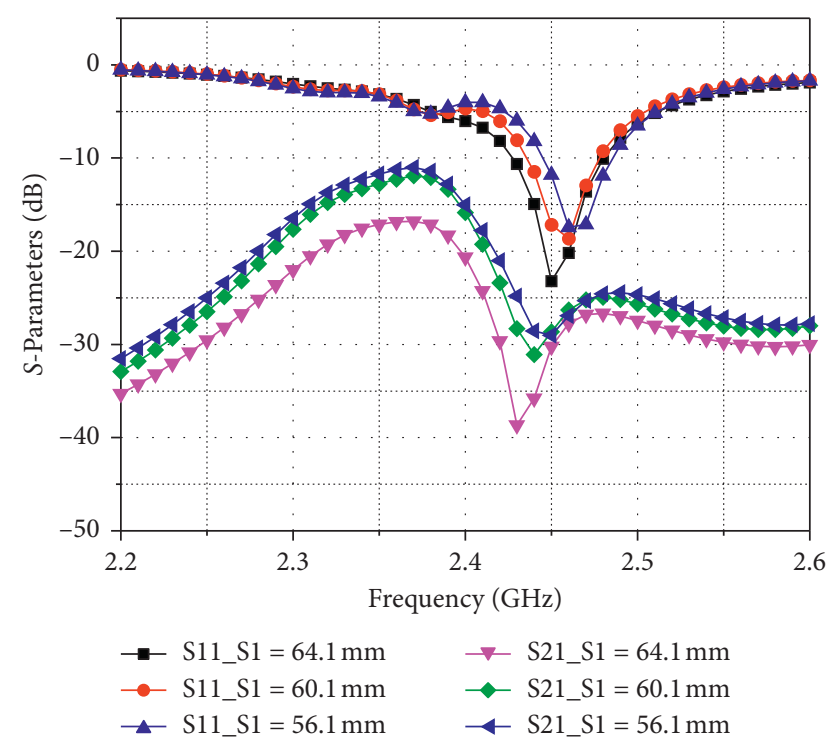

FIgURE 4: Relationship between the $S$-parameter and $S_{1}$.

structure is compared when the center distance of the PIFAs remains $0.5 \lambda_{0}$, as shown in Figure 10. It is observed that the minimum coupling value is reduced by approximately $10 \mathrm{~dB}$, which is less than $-35 \mathrm{~dB}$ in the entire working bandwidth.

\section{Experimental Results and Discussion}

To validate the MC performance of the designed antenna, a prototype is fabricated (Figure 11) and measured in an anechoic chamber. The $S$-parameters and radiation patterns are simulated and measured. The ECC, DG, and TARC are studied to further evaluate the diversity performance.
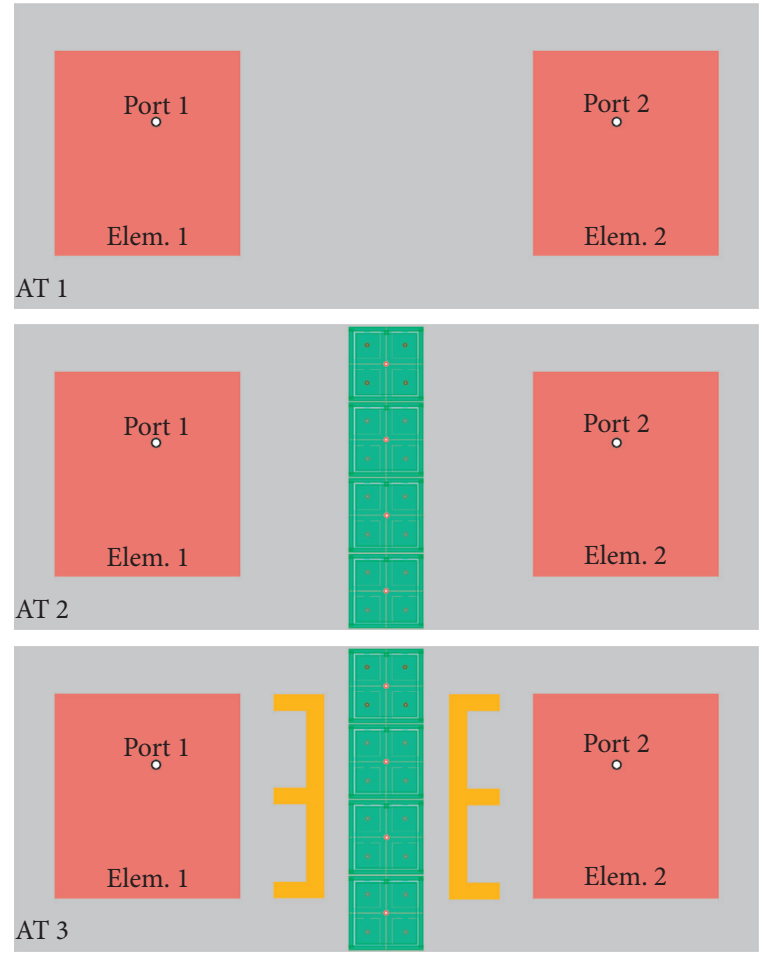

Figure 5: Configurations of Ant 1, Ant 2, and Ant 3.

3.1. Antenna Performance. The S-parameters of the designed antenna are simulated and measured, and the results are illustrated in Figure 12. The measured result of $S_{11}$ shows that the resonant frequency of the antenna is $2.43 \mathrm{GHz}$, which is $0.02 \mathrm{GHz}$ lower than the simulated result. The slight shift in the resonance frequency may be due to common factors, such as the tolerance on the FR4 dielectric constant, inaccuracy in the fabrication process, and effect of the SMA connector. The 


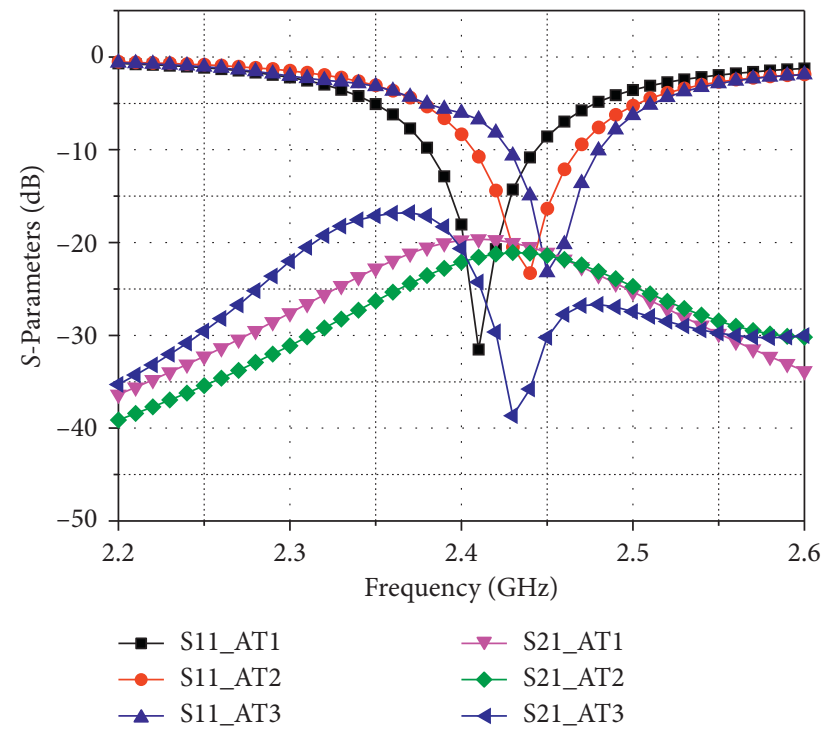

Figure 6: Simulated $S$-parameters for AT1, AT2, and AT3.

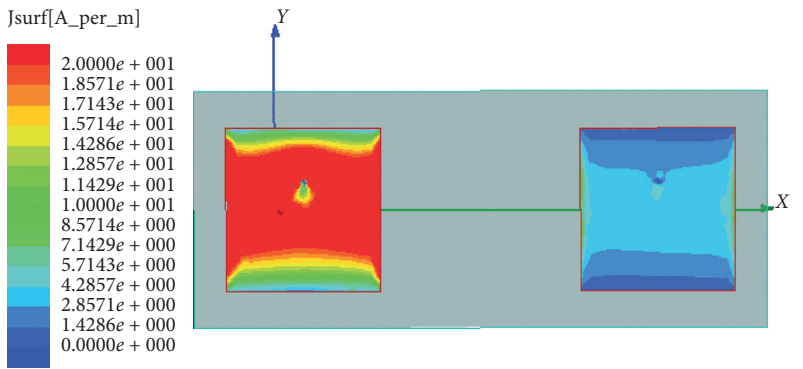

(a)

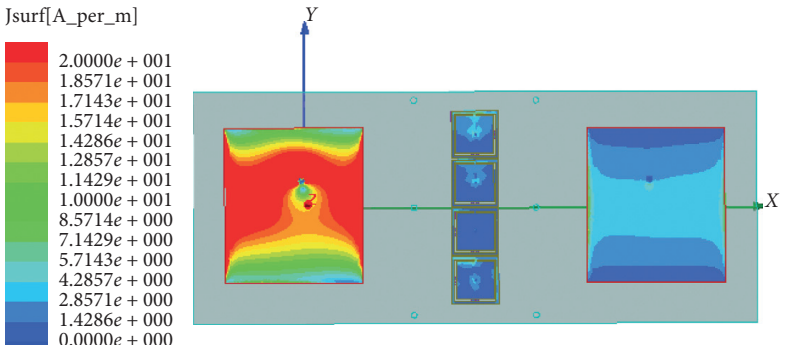

(b)

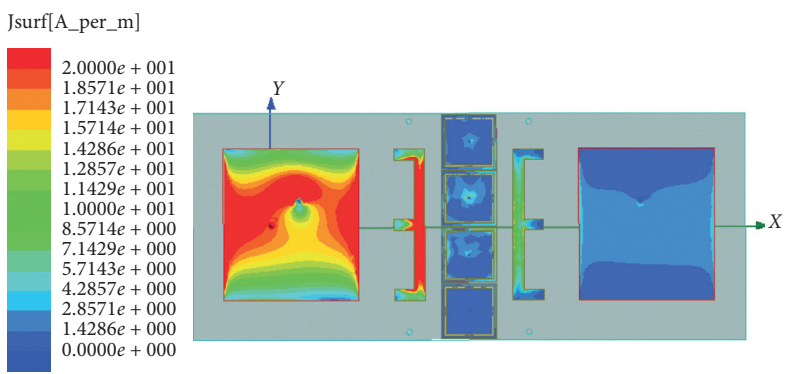

(c)

Figure 7: Surface current distribution: (a) AT1; (b) AT2; (c) AT3.

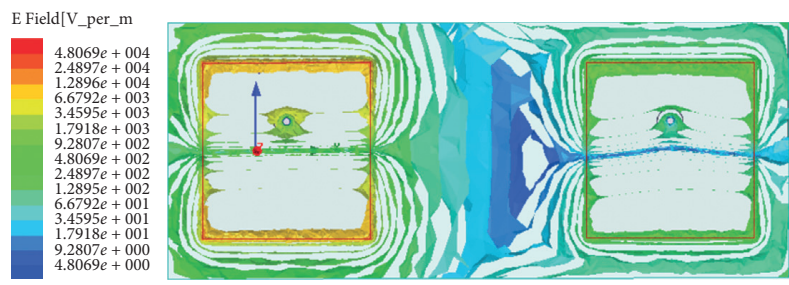

(a)

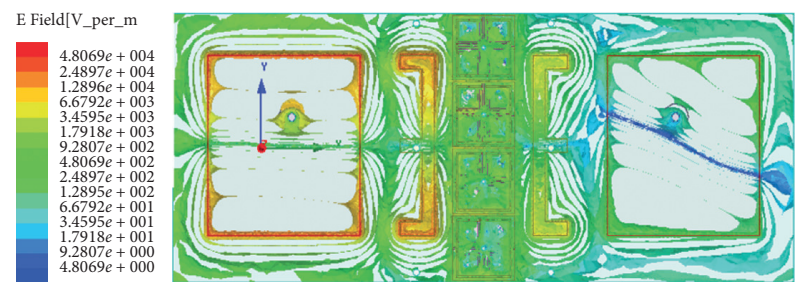

(b)

Figure 8: Electric field distribution: (a) AT1; (b) AT3. 


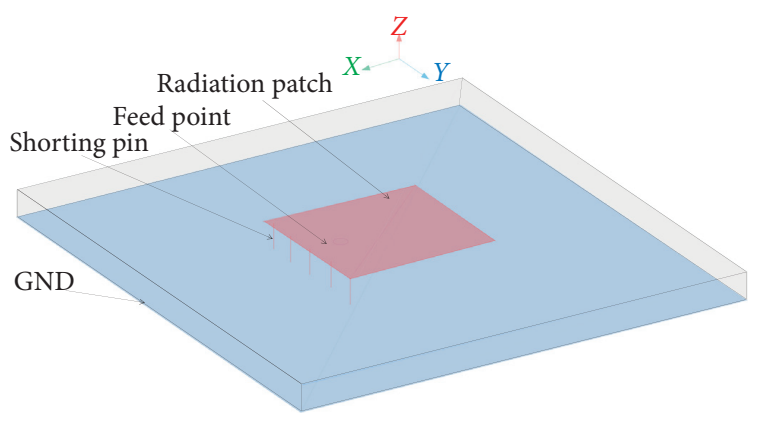

(a)

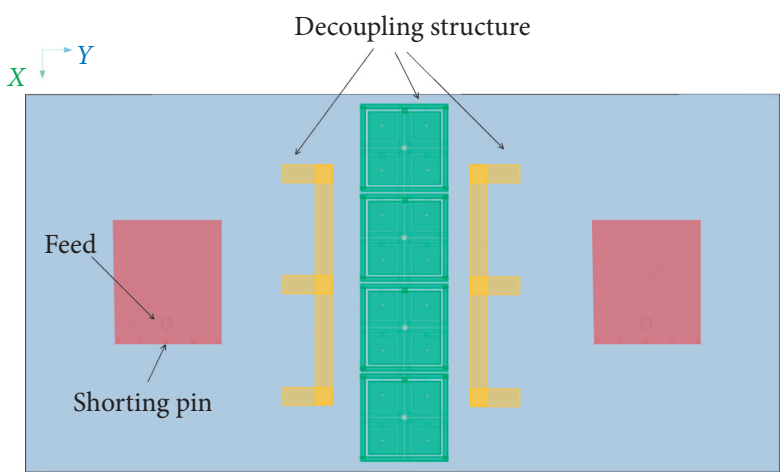

(b)

Figure 9: Geometry of the PIFA (a) antenna unit and (b) two antennas with decoupling structure.

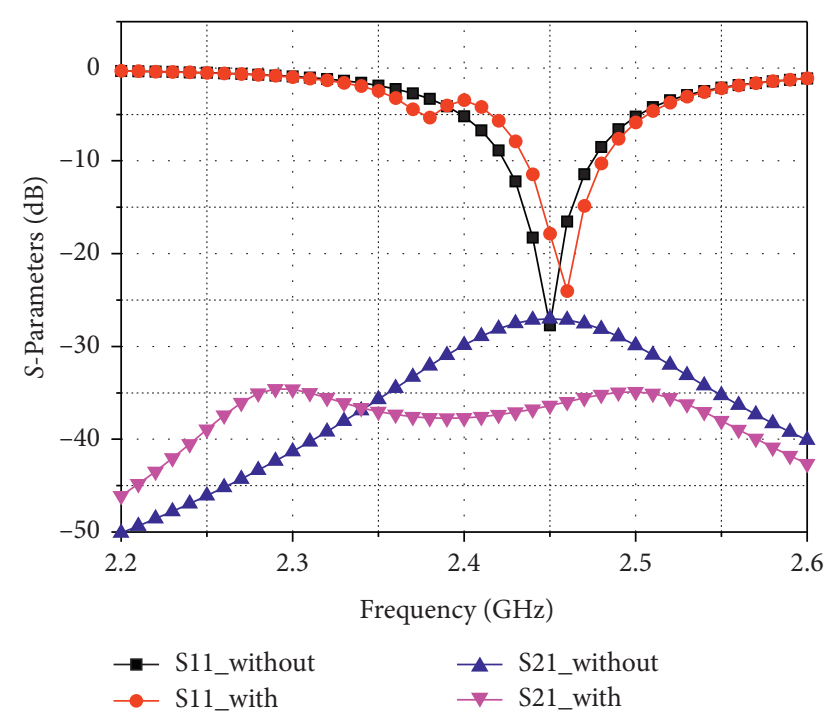

FIgURE 10: Effect of the decoupling structure on the $S$-parameters between two PIFA antennas.

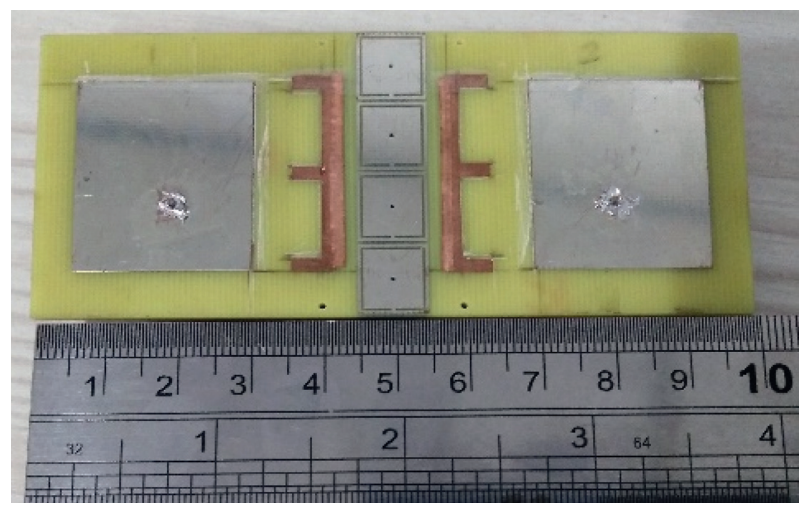

FIgURE 11: Photograph of the fabricated prototype.

simulated $S_{21}$ of the operating frequency is $-38.6 \mathrm{~dB}$, and the measured is $-35 \mathrm{~dB}$. The MC of the proposed antenna is less than $-35 \mathrm{~dB}$ within the operation bandwidth of $-10 \mathrm{~dB}$.

Figure 13 shows the radiation patterns of measured and simulated when one port of the antenna is excited and the

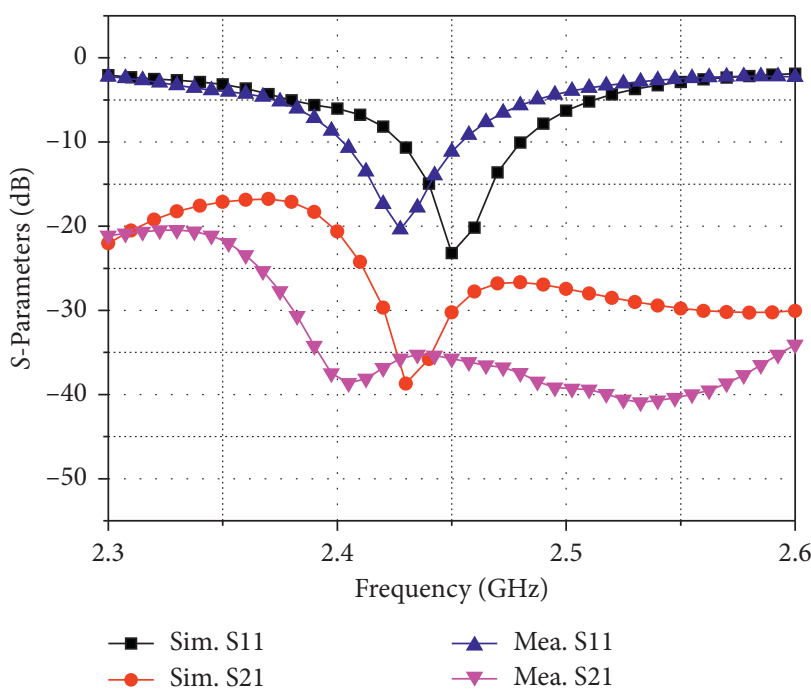

FIGURE 12: Simulated and measured $S$-parameters of the fabricated prototype.

other port is connected to $50 \Omega$ resistance. The measured results correspond with the simulated results. In the main lobe patterns of the upper-sphere space, the simulation and measurement results are basically consistent. Compared with the conventional antenna, the peak of the H-plane pattern of AT3 appears in the broadside direction. The proposed decoupling structure of increasing isolation can effectively reduce the influence of the adjacent element on the main lobe of the pattern. With the insertion of IDLM and E-shaped stub, the cross-polarization level of the antenna is almost unaffected. Within $3 \mathrm{~dB}$ beam width, the relative cross-polarization level is greater than $18 \mathrm{~dB}$.

The simulated and measured gain and radiation efficiency of the AT1 and AT3 are shown in Figure 14. It can be seen that the simulated efficiency of AT1 and AT3 are $47 \%$ and $51 \%$ at $2.45 \mathrm{GHz}$, respectively. When the frequency is higher than $2.45 \mathrm{GHz}$, the gain and efficiency of AT3 are improved because the matching and isolation of AT3 is better than that of AT1 (as shown in Figure 6). The gain and efficiency of AT3 are basically consistent with the simulation results. 

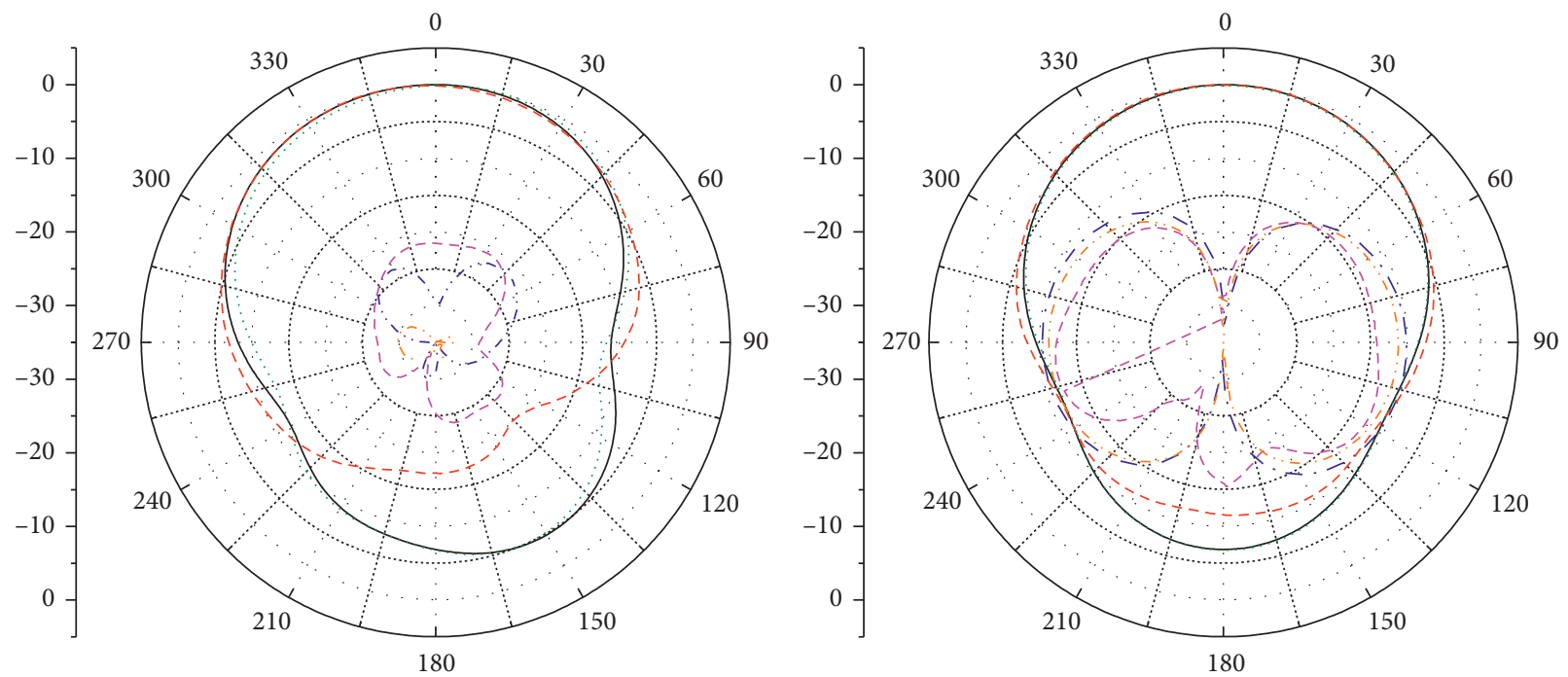

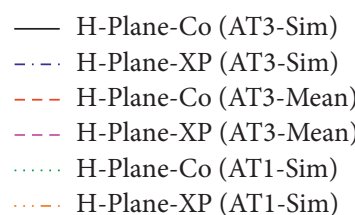

(a)

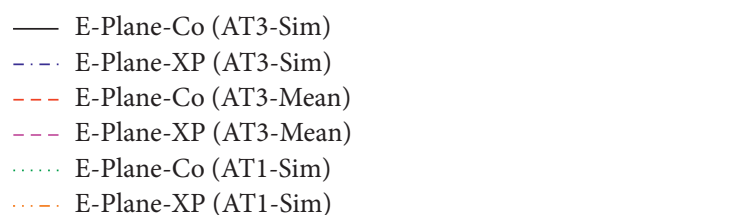

(b)

FIGURE 13: Simulated and measured far-field radiation patterns: (a) $\mathrm{H}$ plane; (b) E plane.

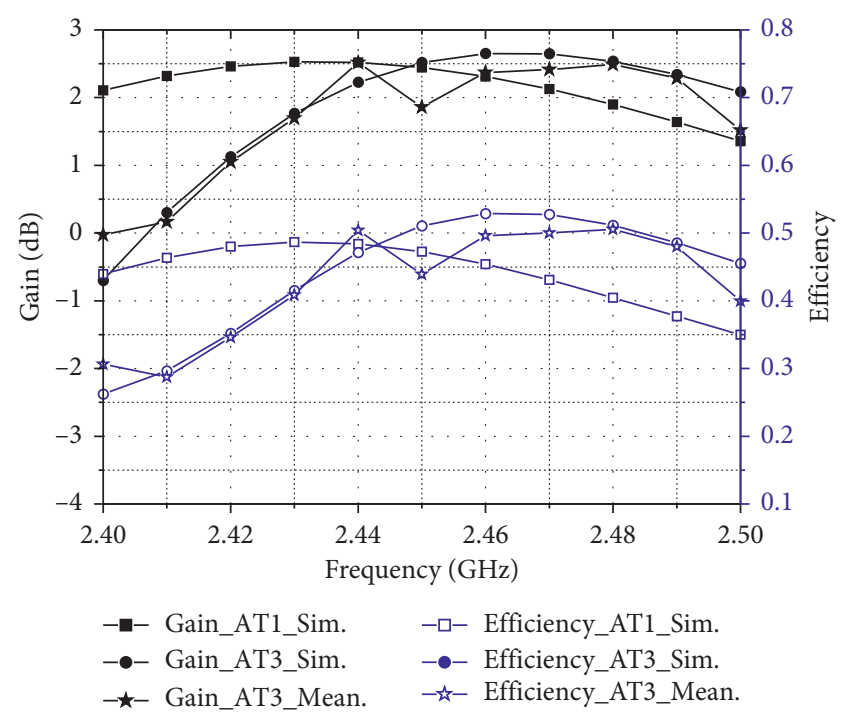

FIgURE 14: Gain and radiation efficiency of AT1 and AT3.

This method is compared with the common mutual coupling suppression methods of microstrip radiation patch, as shown in Table 2. It can be seen that the proposed method of combining the stub and EBG can achieve a better isolation than others with the same center-to-center spacing. Although the antenna in [21] achieves a wider bandwidth and better isolation, it has a higher profile and larger spacing.

3.2. Diversity Analysis. To evaluate the diversity characteristic, the ECC and $D G$ of the designed antenna are estimated.
The ECC can be calculated using $S$-parameters $[14,22]$ or far-field vector phasor radiation patterns $[23,24]$. In [25], the author has verified that the two methods are equivalent in calculating the ECC of half-wave dipoles and orthogonal fed microstrip antennas. Comparing with a radiation pattern method, $S$-parameter method is more convenient in data acquisition and calculation. When the radiation efficiency of the antenna is low, the radiation efficiency should be considered while using $S$-parameter to calculate $E C C[17,26]$ as equation (1), where $S_{a a}$ and $S_{b b}$ represent the reflection coefficients of the two element ports, $S_{a b}$ and $S_{b a}$ represent the transmission coefficients of the two element ports, and $\eta_{a}$ and $\eta_{b}$ are the radiation efficiencies of the two elements:

$$
\text { ECC }=\frac{\left|S_{a a}^{*} S_{a b}+S_{b a}^{*} S_{b b}\right|^{2}}{\left(1-\left|S_{a a}\right|^{2}-\left|S_{b a}\right|^{2}\right)\left(1-\left|S_{b b}\right|^{2}-\left|S_{a b}\right|^{2}\right) \eta_{a} \eta_{b}} .
$$

The DG can be estimated by ECC [17], and the calculation formula is shown as follows:

$$
\mathrm{DG}=10 \sqrt{1-(\mathrm{ECC})^{2}} .
$$

The ECC and DG of the proposed antenna are illustrated in Figure 15. The simulation results are in good agreement with the measured in the range of $2.42-2.48 \mathrm{GHz}$. The estimated value of ECC is far less than the commonly used threshold level of 0.5 , and the value of $D G$ is large, very close to $10 \mathrm{~dB}$. These characteristics show that the designed antenna has good diversity performance and is suitable for $\mathrm{Rx} /$ Tx or MIMO system. 
TABle 2: Dimensions of optimized parameters of the proposed antenna.

\begin{tabular}{lcccccc}
\hline Ref. & Method & $f_{0}(\mathrm{GHz})$ & $\begin{array}{c}\text { Freq. range } \\
\left(\left|\mathrm{S}_{11}\right|<10 \mathrm{~dB}\right)\end{array}$ & Max isolation $(\mathrm{dB})$ & $\begin{array}{c}\text { Center-to-center } \\
\text { spacing }\end{array}$ & Total size $(\mathrm{mm})$ \\
\hline$[19]$ & EBG & 5.1 & $/$ & 30 & $0.5 \lambda_{0}$ & $>0.82 \lambda_{0} \times 0.50 \lambda_{0} \times 0.03 \lambda_{0}$ \\
{$[20]$} & EBG & 5 & $2.4 \%$ & 34 & $0.5 \lambda_{0}$ & $1.3 \lambda_{0} \times 1.0 \lambda_{0} \times 0.02 \lambda_{0}$ \\
{$[21]$} & Decoupling feeding network & 7.5 & $9.2 \%$ & 58 & $0.65 \lambda_{0}$ & $\lambda_{0} \times 1.01 \lambda_{0} \times 0.2 \lambda_{0}$ \\
\hline This work & Stub and EBG & 2.45 & $2.4 \%$ & 39 & $0.5 \lambda_{0}$ & $0.81 \lambda_{0} \times 0.33 \lambda_{0} \times 0.02 \lambda_{0}$ \\
\hline
\end{tabular}

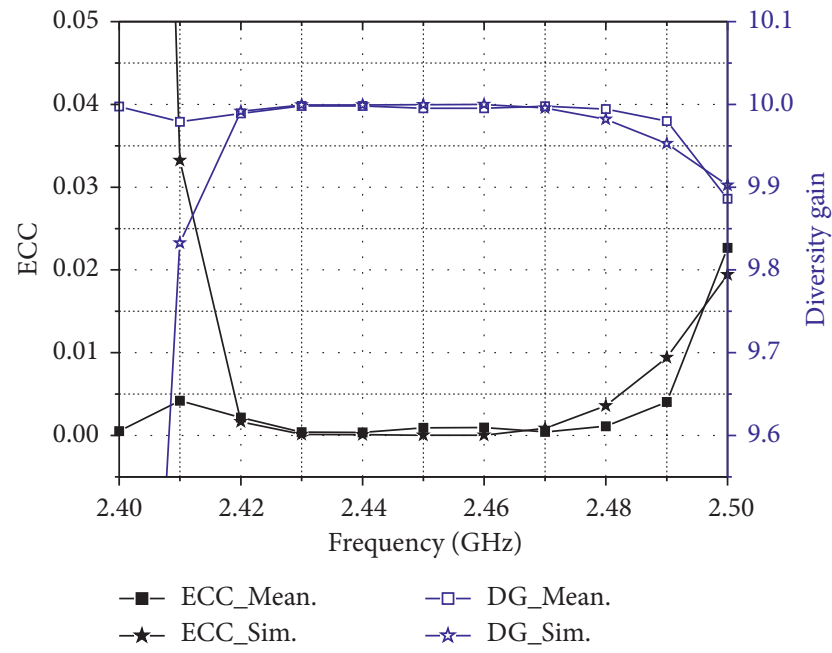

Figure 15: ECC and DG of the proposed antenna.

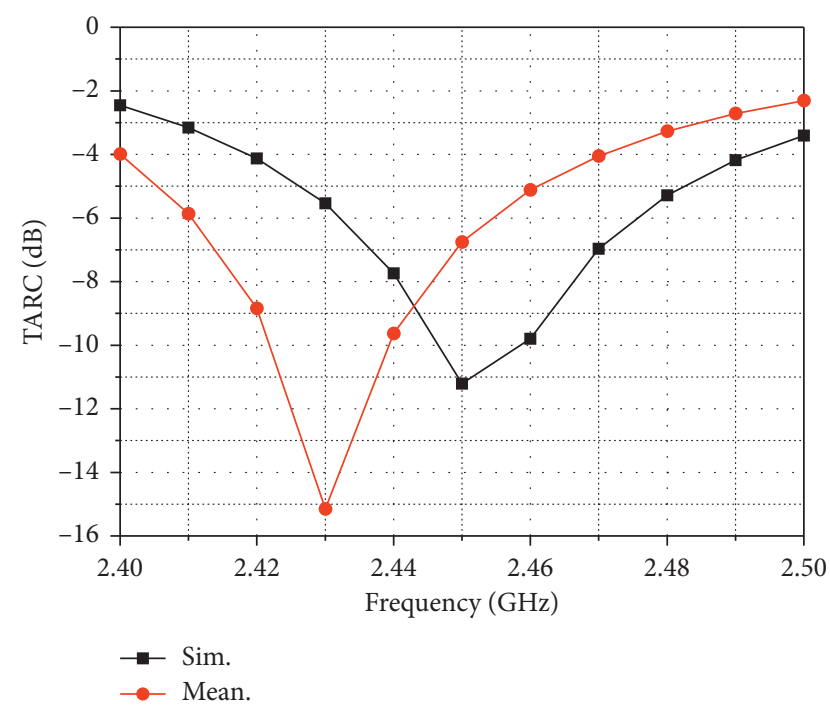

FIGURE 16: Simulated and measured TARC of the proposed antenna.

Total active reflection coefficient (TARC) is another important parameter to evaluate the diversity performance. The TARC can be calculated by equation (3) [27]. When TARC is less than $0 \mathrm{~dB}$, MIMO system can work normally [17]. As shown in Figure 16, the simulated and measured TARC are $-6 \mathrm{~dB}$ and $-11 \mathrm{~dB}$ for the desired frequency $2.45 \mathrm{GHz}$ :

$$
\text { TARC }=\sqrt{\frac{\left|\left(S_{a a}+S_{a b}\right)\right|^{2}+\left|\left(S_{b a}+S_{b b}\right)\right|^{2}}{2}} .
$$

\section{Conclusion}

An efficient technique for adding IDLM and back-to-back E-shaped stubs is proposed for MC suppression in microstrip patch arrays with center-to-center spacing between antenna elements of $0.5 \lambda_{0}$. The designed IDLM and E-shaped stub are placed between the $\mathrm{H}$-coupled radiation elements. The influence of coupling elements on the coupling and radiation performance is discussed, and the reason is analyzed through the current and electric field on the antenna. Moreover, the ECC, DG, and TARC of the proposed structure are also studied. The ECC is very small, the $D G$ is large, and the TARC is less than $-3 \mathrm{~dB}$. Owing to the abovementioned features, the proposed structure is a promising candidate for the wireless $\mathrm{Rx} / \mathrm{Tx}$ or $\mathrm{MIMO}$ applications.

\section{Data Availability}

The design results can be reproduced using Figure land Table 1 of the paper

\section{Conflicts of Interest}

The authors declare that they have no conflicts of interest.

\section{Acknowledgments}

This work was supported in part by the Project of Guangxi Youth Innovative Talents Research (no. 2019AC20281), in part by the Project of Guangxi Natural Science Foundation (no. 2019JJA170029), and in part by Guangxi Key Laboratory of Wireless Wideband Communication and Signal Processing, Guilin University of Electronic Technology (no. GXKL06190201).

\section{References}

[1] T. Shabbir, R. Saleem, S. S. Al-bawri, M. F. Shafique, and M. T. Islam, "Eight-port metamaterial loaded UWB-MIMO antenna system for 3D system-in-package applications," IEEE Access, vol. 8, pp. 106982-106992, 2020.

[2] S. Ebadi and A. Semnani, "Mutual coupling reduction in waveguide-slot-array antennas using electromagnetic bandgap (EBG) structures," IEEE Antennas and Propagation Magazine, vol. 56, no. 3, pp. 68-79, 2014.

[3] J. Ouyang, F. Yang, and Z. M. Wang, "Reducing mutual coupling of closely spaced microstrip MIMO antennas for WLAN application," IEEE Antennas and Wireless Propagation Letters, vol. 10, pp. 310-313, 2011.

[4] M. Li, B. G. Zhong, and S. W. Cheung, "Isolation enhancement for MIMO patch antennas using near-field resonators as 
coupling-mode transducers," IEEE Transactions on Antennas and Propagation, vol. 67, no. 2, pp. 755-764, 2019.

[5] B. L. Dhevi, K. S. Vishvaksenan, and K. Rajakani, "Isolation enhancement in dual-band microstrip antenna array using asymmetric loop resonator," IEEE Antennas and Wireless Propagation Letters, vol. 17, no. 2, pp. 238-241, 2018.

[6] X. Tang, X. Qing, and Z. N. Chen, "Simplification and implementation of decoupling and matching network with port pattern-shaping capability for two closely spaced antennas," IEEE Transactions on Antennas and Propagation, vol. 63, no. 8, pp. 3695-3699, 2015.

[7] C.-H. Wu, C.-L. Chiu, and T.-G. Ma, "Very compact fully lumped decoupling network for a coupled two-element array," IEEE Antennas and Wireless Propagation Letters, vol. 15, pp. 158-161, 2016.

[8] K. S. Vishvaksenan, K. Mithra, R. Kalaiarasan, and K. S. Raj, "Mutual coupling reduction in microstrip patch antenna arrays using parallel coupled-line resonators," IEEE Antennas and Wireless Propagation Letters, vol. 16, pp. 2146-2149, 2017.

[9] A. Habashi, J. Nourinia, and C. Ghobadi, "Mutual coupling reduction between very closely spaced patch antennas using lowprofile folded split-ring resonators (FSRRs)," IEEE Antennas and Wireless Propagation Letters, vol. 10, pp. 862-865, 2011.

[10] K. Wei, J.-Y. Li, L. Wang, Z.-J. Xing, and R. Xu, "Mutual coupling reduction by novel fractal defected ground structure bandgap filter," IEEE Transactions on Antennas and Propagation, vol. 64, no. 10, pp. 4328-4335, 2016.

[11] Y.-F. Cheng, X. Ding, W. Shao, and B.-Z. Wang, "Reduction of mutual coupling between patch antennas using a polarization-conversion isolator," IEEE Antennas and Wireless Propagation Letters, vol. 16, pp. 1257-1260, 2017.

[12] J.-Y. Lee, S.-H. Kim, and J.-H. Jang, "Reduction of mutual coupling in planar multiple antenna by using 1-D EBG and SRR structures," IEEE Transactions on Antennas and Propagation, vol. 63, no. 9, pp. 4194-4198, 2015.

[13] B. Mohamadzade and M. Afsahi, "Mutual coupling reduction and gain enhancement in patch array antenna using a planar compact electromagnetic bandgap structure," IET Microwaves, Antennas \& Propagation, vol. 11, no. 12, pp. 1719-1725, 2017.

[14] A. H. Radhi, R. Nilavalan, Y. Wang, H. Al-Raweshidy, A. A. Eltokhy, and N. A. Aziz, "Mutual coupling reduction with a novel fractal electromagnetic bandgap structure," IET Microwaves, Antennas \& Propagation, vol. 13, no. 2, pp. 134-141, 2019.

[15] M. F. Shafique, Z. Qamar, L. Riaz, R. Saleem, and S. A. Khan, "Coupling suppression in densely packed microstrip arrays using metamaterial structure," Microwave and Optical Technology Letters, vol. 57, no. 3, pp. 759-763, 2015.

[16] R. Hafezifard, M. N. Moghadasi, J. R. Mohassel, and R. A. Sadeghzadeh, "Mutual coupling reduction for two closely spaced meander line antennas using metamaterial substrate," IEEE Antennas Wireless Propag. Lett., vol. 15, pp. 40-43, 2016.

[17] P. Garg and P. Jain, "Isolation improvement of MIMO antenna using a novel flower shaped metamaterial absorber at $5.5 \mathrm{GHz}$ WiMAX band," IEEE Transactions on Circuits and Systems II: Express Briefs, vol. 67, no. 4, pp. 675-679, 2020.

[18] S. Ghosh, T.-N. Tran, and T. Le-Ngoc, "Dual-layer EBG-based miniaturized multi-element antenna for MIMO Systems," IEEE Transactions on Antennas and Propagation, vol. 62, no. 8, pp. 3985-3997, 2014.

[19] Q. L. Zhang, Y. T. Jin, J. Q. Feng, X. Lv, and L. M. Si, “Mutual coupling reduction of microstrip antenna array using metamaterial absorber," in Proceedings of the IEEE MTT-S International Microwave Workshop Series on Advanced Materials and Processes for RF and THz Applications (IMWSAMP 2020), pp. 1-3, Suzhou, China, July 2015.

[20] M. M. Bait-Suwailam, O. F. Siddiqui, and O. M. Ramahi, "Mutual coupling reduction between microstrip patch antennas using slotted-complementary split-ring resonators," IEEE Antennas and Wireless Propagation Letters, vol. 9, pp. 876-878, 2010.

[21] R.-L. Xia, S.-W. Qu, P.-F. Li, Q. Jiang, and Z.-P. Nie, “An efficient decoupling feeding network for microstrip antenna array," IEEE Antennas and Wireless Propagation Letters, vol. 14, pp. 871-874, 2015.

[22] P. Gao, S. He, X. Wei, Z. Xu, N. Wang, and Y. Zheng, "Compact printed UWB diversity slot antenna with $5.5-\mathrm{GHz}$ band-notched characteristics," IEEE Antennas and Wireless Propagation Letters, vol. 13, pp. 376-379, 2014.

[23] M. Li, L. Jiang, and K. L. Yeung, "Novel and efficient parasitic decoupling network for closely coupled antennas," IEEE Transactions on Antennas and Propagation, vol. 67, no. 6, pp. 3574-3585, 2019.

[24] M. Li, L. Jiang, and K. L. Yeung, "A novel wideband decoupling network for two antennas based on the Wilkinson power divider," IEEE Transactions on Antennas and Propagation, vol. 68, no. 7, pp. 5082-5094, 2020.

[25] S. Blanch, J. Romeu, and I. Corbella, "Exact representation of antenna system diversity performance from input parameter description," Electronics Letters, vol. 39, no. 9, pp. 705-707, 2003.

[26] S. A. Ja'afreh, Y. Huang, and L. Xing, “"Low profile and wideband planar inverted-F antenna with polarization and pattern diversities," IET Microw," Antennas Propag, vol. 10, no. 2, pp. 152-161, 2016.

[27] S. h. Chae, W.i. Kawk, S. Park, and K. Lee, "Analysis of mutual coupling in MIMO antenna Array by TARC calculation," in Proceedings of the 2006 Asia-Pacific Microwave Conference, pp. 2090-2093, Pacifico, Yokohama, December 2006. 